\title{
The phantom ligand effect: allosteric control of transcription by the retinoid $\mathrm{X}$ receptor
}

\author{
Ira G. Schulman, ${ }^{1,3}$ Chuan Li, ${ }^{1,2}$ John W.R. Schwabe, ${ }^{1}$ and Ronald M. Evans ${ }^{1,2,4}$ \\ ${ }^{1}$ The Salk Institute for Biological Sciences and ${ }^{2}$ The Howard Hughes Medical Institute, La Jolla, California 92037 USA
}

\begin{abstract}
Regulation of gene expression via allosteric control of transcription is one of the fundamental concepts of molecular biology. Studies in prokaryotes have illustrated that binding of small molecules or ligands to sequence-specific transcription factors can produce conformational changes at a distance from the binding site. These ligand-induced changes can dramatically alter the DNA binding and/or trans-activation abilities of the target transcription factors. In this work, analysis of trans-activation by members of the steroid and thyroid hormone receptor superfamily identifies a unique form of allosteric control, the phantom ligand effect. Binding of a novel ligand (LG100754) to one subunit (RXR) of a heterodimeric transcription factor results in a linked conformational change in the second noncovalently bound subunit of the heterodimer (RAR). This conformational change results in both the dissociation of corepressors and association of coactivators in a fashion mediated by the activation function of the non-liganded subunit. Without occupying the RAR hormone binding pocket, binding of LG100754 to RXR mimics exactly the effects observed when hormone is bound to RAR. Thus, LG100754 behaves as a phantom ligand.
\end{abstract}

[Key Words: Transcription; RXR; phantom ligand effect; RAR]

Received October 8, 1996; revised version accepted December 17, 1996.

Nuclear receptors are hormone-activated transcription factors that regulate complex gene networks influencing vertebrate development, differentiation, and homeostasis. Recent crystallographic analyses of several receptors confirm earlier work suggesting that hormone induces a significant structural change in the nuclear receptor ligand-binding domain (Bourguet et al. 1995; Renaud et al. 1995; Wagner et al. 1995). This induced structural transition is thought to allow receptors to assume a conformation favoring interactions with positive-acting cofactors. Nevertheless, how hormone-induced conformational changes are transduced into a molecular signal that activates transcription remains a mystery /for review, see Kastner et al. 1995; Mangelsdorf and Evans 1995; Mangelsdorf et al. 1995).

Receptors for thyroid hormone (TR), retinoic acid (RAR), vitamin D (VDR), prostanoids (PEAR), and numerous orphan receptors preferentially bind to DNA as heterodimers with a common partner, the retinoid $\mathrm{X}$ receptor (RXR). RXRs can also bind to DNA as homodimers and activate transcription in response to 9-cis retinoic acid. The requirement for the common RXR partner in a diverse array of hormonal signaling path-

\footnotetext{
${ }^{3}$ Present address: Department of Retinoid Research, Ligand Pharmaceuticals, San Diego, California 92121 USA.

${ }^{4}$ Corresponding author.

E-MAIL Evans@salk.edu; FAX (619) 455-1349.
}

ways illustrates the critical role of RXRs in hormonedependent gene regulation (for review, see Mangelsdorf and Evans, 1995).

That RXR can bind and respond to 9-cis retinoic acid as a homodimer and participate as a heterodimeric partner with multiple nuclear receptors, presents a quandary. Does 9-cis retinoic acid influence all heterodimeric signaling regardless of the partner? Recent work has identified two classes of RXR-dependent heterodimers. There are permissive heterodimers that allow RXR signaling (e.g., RXR-PPAR, RXR-LXR, RXR-NGFIB) and nonpermissive heterodimers that can inhibit signaling by RXR (e.g., RXR-RAR, RXR-TR; for review, see Leblanc and Stunnenberg 1995). When dimerized with a nonpermissive partner, RXR signaling is blocked by two mechanisms. First, the partner recruits the recently identified class of thyroid and retinoid corepressors (TRACs; Chen and Evans 1995; Horlein et al. 1995; Kurokawa et al. 1995). Second, dimerization with nonpermissive partners significantly decreases the ability of RXR to bind hormone (Kurokawa et al. 1994; Forman et al. 1995). This ability of the partner to modulate RXR signaling provides cells with a mechanism to restrict the activity of RXR to specific hormonal signaling pathways. From these observations, it has been suggested that RXR acts as a silent partner in nonpermissive heterodimers.

Although signaling by RXR in response to ligand is inhibited in nonpermissive heterodimers, whether RXR contributes to the transcriptional activity of the complex 
remains unclear. Dimerization with RXR enhances trans-activation by TR (Claret et al. 1996) and deletion of the RXR hormone-dependent activation function $(\tau \mathrm{c}$, AF-2) significantly reduces hormone-activated transcription by the partner (Leng et al. 1995; Schulman et al. 1996). We have shown recently that the ability of the $\mathrm{RXR} \tau c$ domain to affect partner-activated transcription is mediated by the ability of RXR to influence interaction with corepressors (Schulman et al. 1996). In addition, the identification of a novel RXR-specific ligand, LG100754, which leads to activation of transcription by nonpermissive RXR-RAR heterodimers (Canan Koch et al. 1996; Lala et al. 1996) supports the idea that RXR can participate in signaling even in nonpermissive complexes.

In this work, we address how LG100754 binding to RXR leads to trans-activation by nonpermissive RXRRAR heterodimers. In contrast to other RXR-specific ligands, LG100754 binds to RXR when present in a RXRRAR heterodimer (Canan Koch et al. 1996; Lala et al. 1996). Thus, binding of LG100754 may simply lead to trans-activation mediated by the RXR $\tau c$ domain in much the same way that all-trans retinoic acid leads to trans-activation through RAR. In the present study, however, a combination of trans-activation and repression defective receptors are used to show that, unexpectedly, binding of LG100754 to RXR leads to trans-activation mediated by the hormone-dependent activation function ( $\tau$ c domain, AF-2) of RAR. Thus, even though RAR remains non-liganded, it behaves as if ligand is bound. This unique form of hormonal signaling in which ligand binding by one member of the heterodimer leads to activation of transcription by the second non-liganded member of the pair has been termed the phantom ligand effect. The results presented here indicate an intimate relationship between the heterodimeric partners and suggest that the allosteric changes required for receptor activation may not require direct ligand binding.

\section{Results}

Activation by LG100754 is mediated by RAR

Analysis of receptor-specific ligands has supported the idea that RXR cannot respond to hormone when dimerized with a non-liganded nonpermissive partner such as RAR (Kurokawa et al. 1994; Forman et al. 1995). Therefore, the ability of the RXR-specific antagonist LG100754 to stimulate RXR-RAR heterodimers was unexpected (Canan Koch et al. 1996; Lala et al. 1996). These paradoxical properties of LG100754 are illustrated in Figure 1 by use of a GAL4-based assay. As expected, the RXR-specific agonist LGD1069 activates a GAL4-RXR ligand-binding domain fusion (GAL4-RXRLBD) whereas the RAR-specific agonist AM580 has no effect (Fig. 1A). This response profile is reversed when a plasmid expressing the RAR ligand binding domain is cotransfected along with GAL4-RXRLBD (Fig. 1B). The results of Figure 1 illustrate the ability of RAR to suppress hormonal signaling by RXR-specific agonists (e.g., LGD1069) while still allowing the heterodimer to respond to RAR-specific agonists. As expected, the RXR-specific antagonist LG100754 fails to activate GAL4-RXRLBD alone (Fig. 1A\}. As shown in Figure 1B, however, LG100754 produces $\sim 10$-fold activation in the context of the RXRRAR heterodimer. The ability of LG100754 to activate transcription appears to be relatively heterodimer specific. Although LG100754 activates RXR-PPAR heterodimers (Lala et al. 1996), it does not activate RXR-TR, -VDR, -LXR, or -NGFIB heterodimers (data not shown). LG100754 has also been shown to activate RAR responsive pathways in vivo such as the differentiation of the acute promyelocytic leukemia cell line NB4 (Lala et al. 1996; data not shown) indicating that the unusual activation profile seen with this ligand is not an artifact of the GAL4 system.

In ligand-binding experiments, LG100754 has been shown to bind to RXRs with nanomolar affinity but has only very weak affinity for RARs ( $k D>1.0 \mu \mathrm{M}$ ) (Canan Koch et al. 1996; Lala et al. 1996). Those binding experiments, however, examined the ability of LG100754 to bind to isolated subunits of the heterodimer and could not rule out the possibility that LG100754 binds with high affinity to RAR when it is present in a RXR-RAR heterodimer. To address the specificity of LG100754 binding to RXR-RAR heterodimers, we expressed and purified a recombinant heterodimer comprised of the ligand binding domains of human $\operatorname{RXR} \alpha$ and human RAR $\alpha$ (see Materials and Methods). To examine binding of LG100754 to purified heterodimers, the competitive binding experiment shown in Figure $2 \mathrm{~A}$ was carried out. Purified heterodimers were allowed to bind ${ }^{3} \mathrm{H}$ all-trans retinoic acid (a RAR-specific ligand; Allegretto et al. 1993; Allenby et al. 1993) in the presence or absence of a 1000-fold excess of unlabeled competitor. The heterodimeric complex was then recovered by use of the 6-histidine tag on the RAR subunit. As expected, binding of ${ }^{3} \mathrm{H}$ all-trans retinoic acid is reduced to background levels $(\sim 50 \mathrm{cpm})$ by excess unlabeled all-trans retinoic acid. The RXR-specific compounds LGD1069 and LG100754, however, do not compete for binding to RAR, confirming their specificity for the RXR subunit of the heterodimer. SDS gel analysis of the $\mathrm{Ni}^{+2}-\mathrm{NTA}$ agarose recovered protein, before and after ligand binding, indicates that the recombinant heterodimers do not dissociate during the binding experiment (Fig. 2B). The results of the binding experiment (Fig. 2), along with the finding that LG100754 is not significantly metabolized in vivo, indicates that activation of RXR-RAR heterodimers by LG100754 is mediated by binding of this compound to RXR.

The unexpected ability of LG100754 to activate nonpermissive RXR-RAR heterodimers suggested a possible role reversal in which $R A R$, instead of $R X R$, functions as the silent partner in the complex. To examine the role of the RXR hormone-activation function $(\tau c, A F-2)$ in this response, plasmids individually expressing wild-type or mutant GAL4-RXR LBD fusions were transfected into CV1 cells. This system evaluates the consequence of mutations in the RXR $\tau$ c domain on activation by RXR 

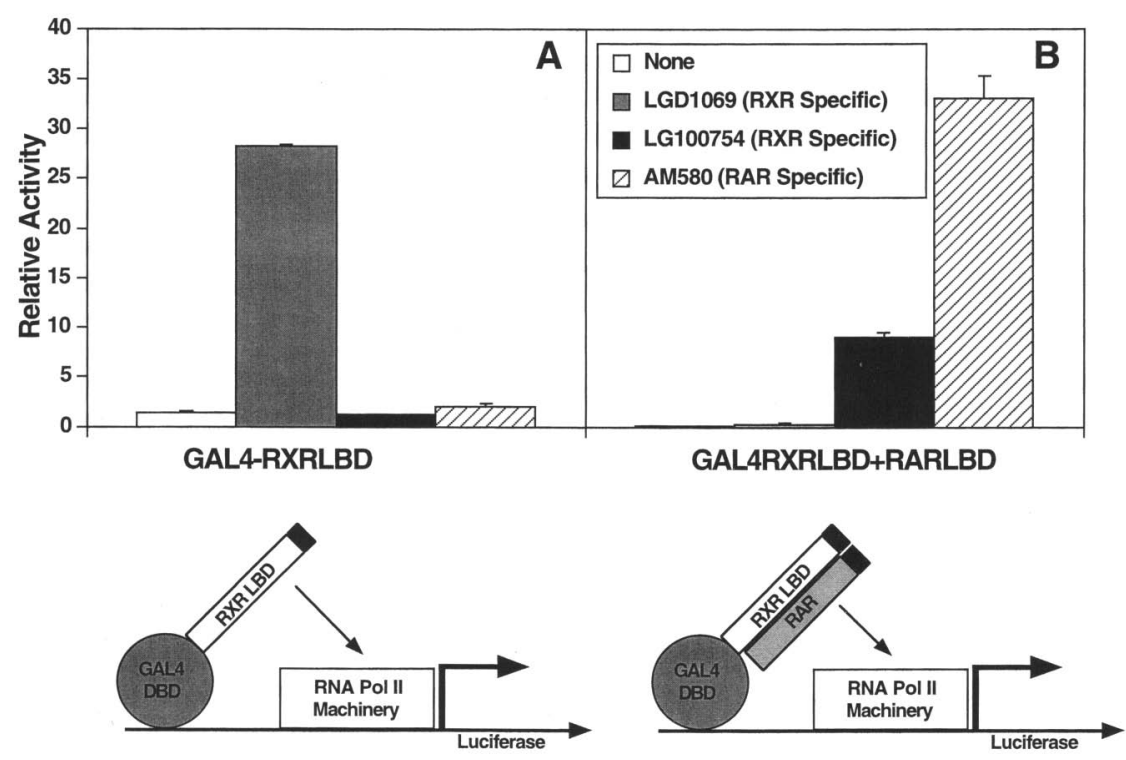

Figure 1. Activity of receptor-specific ligands. A fusion between the DNA-binding domain of GAL4 and RXR ligand-binding domain (amino acids 197-462) was transfected into CV1 cells by itself $(A)$ or along with $(B)$ a construct expressing the ligand binding domain of human $\operatorname{RAR} \alpha$. A reporter with 4 GAL4 binding sites $\left(\mathrm{UAS}_{\mathrm{G}} \times 4\right)$ was included in each case. After transfection, CV1 cells were cultured in the absence (open bars) or presence of $100 \mathrm{nM}$ LGD1069 (shaded bars), $100 \mathrm{~nm}$ LG100754 (solid bars) or $100 \mathrm{nM}$ AM580 (hatched bars) for $36 \mathrm{hr}$. Activity relative to the $\mathrm{UAS}_{\mathrm{G}} \times 4$ luciferase reporter alone is reported. Transfections were normalized by cotransfection with a $\beta$-galactosidase expression plasmid (see Materials and Methods). homodimers or RXR-RAR heterodimers without complications from endogenous RXRs. As expected, the RXR $\tau \mathrm{c}$ domain mutations eliminate trans-activation by RXR homodimers in response to LGD1069 (Fig. 3A and Schulman et al. 1995) revealing the key role for this domain in receptor function. These mutations have little or no effect on the ability of RXR to bind 9-cis retinoic acid or LGD1069 (Schulman et al. 1995; data not shown). Like wild-type RXR, the RXR $\tau$ c domain mutants also do not activate transcription in response to LG100754 (Fig. 3A). Surprisingly, in the context of the heterodimer, this same set of mutations has little or no effect on the fold induction by LG100754 (+ligand/-ligand; Fig. 3B). The approximate twofold decrease in fold induction observed when the RXR $\tau c$ domain is deleted (RXR443), results from increased binding of trans-acting corepressors (Schulman et al. 1996). Results with full-length receptors and a natural response element confirm the faithfulness of the GAL4-fusion based assay (Fig. 3C). These surprising results suggest that even though LG100754 selectively targets RXR, its $\tau \mathrm{c}$ domain is not required for the response.

The above experiments imply that trans-activation by LG100754 may be dependent on the $\tau c$ domain of RAR even though RAR remains unoccupied. This possibility was investigated by determining the ability of a RAR $\tau \mathrm{c}$ domain deletion mutant (RAR403) to respond to LG100754. Indeed, deletion of the RAR $\tau c$ domain completely blocks the activation of RXR-RAR by LG100754 or AM580 (Fig. 4; cf. A with C). Thus, ligands occupying either subunit of the heterodimer appear to act through the RAR $\tau \mathrm{c}$ domain.

Previous work has shown that deletion of the RAR $\tau \mathrm{c}$ domain produces a dominant negative receptor (Baniahmad et al. 1992; Damm et al. 1993; Tate et al. 1994; Tate and Grippo 1995). The dominant negative activity of this mutant receptor (RAR403) results from the constitutive binding of corepressors (Chen and Evans 1995; Kurokawa et al. 1995). To rule out the possibility that the constitutive binding of corepressors to RAR403 may be masking a response to LG100754, a triple point mutation was introduced into the hinge region of the RAR ligand binding domain. This triple mutant in the repressor interacting domain (the CoR-box) has been shown to significantly reduce corepressor binding in vitro and repression of hormone-independent transcription in vivo (data not shown; Hörlein et al. 1995; Kurokawa et al. 1995). When the CoR-box mutation is introduced into the context of RAR403, repression of hormone-independent transcription measured on the DR $5 \times 2$-luciferase reporter is reduced (Fig. 4D). Nevertheless, a response to either LG100754 or AM580 is not restored. In contrast, wildtype RAR with the CoR-box mutation retains the response to both LG100754 and AM580 (B). Thus, the analyses of RAR mutants in Figure 4 supports the proposal that binding of LG100754 to RXR results in transactivation mediated by the $\tau c$ domain of RAR.

\section{LG100754 stimulates interaction with coactivators and release of corepressors}

Recent structural analyses suggest that hormone triggers a folding back of the $\tau c$ domain toward the ligand-binding domain core (Bourguet et al. 1995; Renaud et al. 1995; Wagner et al. 1995). This ligand-induced movement of the $\tau \mathrm{c}$ domain is thought to promote both the release of corepressors and interactions with coactivators. As described above, deletion of the $\tau c$ domain produces receptors that bind ligand but fail to release corepressors (Chen and Evans 1995; Horlein et al. 1995; Kurokawa et al. 1995; Schulman et al. 1996). Likewise, deletion or mutation of the $\tau c$ domain disrupts liganddependent interactions with coactivators /Cavailles et al. 1994; Halachmi et al. 1994; Lee et al. 1995; Schulman et al. 1995; Chakravarti et al. 1996; Kamei et al. 1996; vom Baur et al. 1996). To determine whether LG100754 bind- 


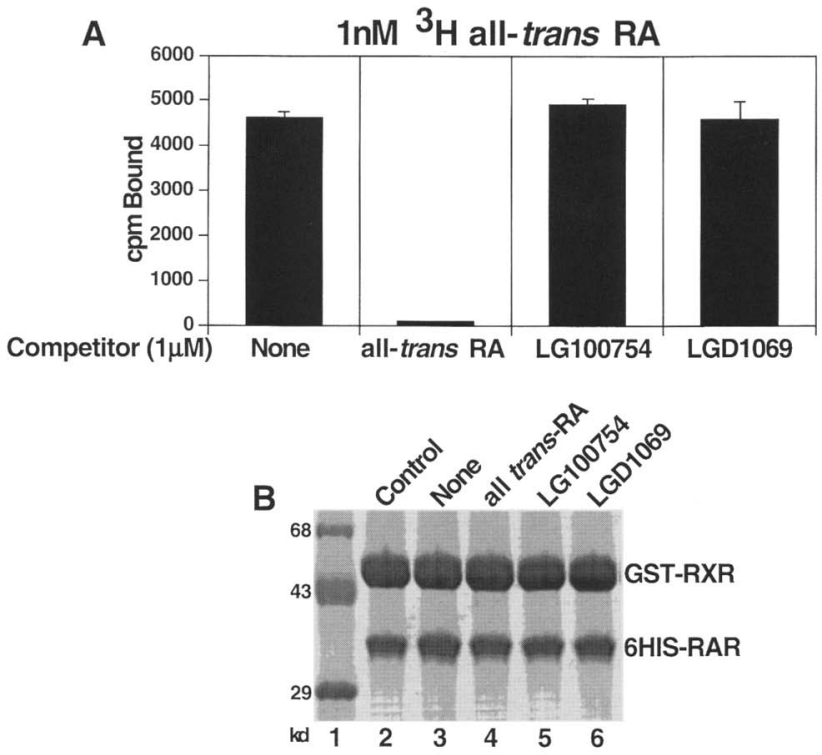

Figure 2. Specific binding of LG100754 to the RXR subunit of RXR-RAR heterodimers. (A) Purified recombinant heterodimers comprised of the ligand-binding domains of human $\operatorname{RXR} \alpha$ and human RAR $\alpha$ were incubated for $60 \mathrm{~min}$ in presence of $1 \mathrm{nM}^{3} \mathrm{H}$ all-trans retinoic acid and a 1000-fold excess $(1.0 \mu \mathrm{M})$ of unlabeled all-trans retinoic acid (RAR-specific), LG100754 (RXR-specific), or LGD1069 (RXR-specific). After ligand binding, the recombinant heterodimers were precipitated with $\mathrm{Ni}^{+2}$. NTA agarose that binds the 6 histidine tag introduced at the amino termini of the RAR ligand-binding domain. After washing the beads, the amount of ${ }^{3} \mathrm{H}$ all-trans retinoic acid bound to the heterodimers was determined by scintillation counting. In the absence of recombinant heterodimer $<50 \mathrm{cpm}$ are bound to the agarose beads. Each sample was analyzed in duplicate. The data presented is the mean + standard error of two independent experiments. $(B)$ Samples treated as in $A$ were eluted from $\mathrm{Ni}^{+2} \mathrm{NTA}$ agarose and analyzed by SDS gel electrophoresis. The apparent excess of GST-RXR to 6 histidine-RAR results from increased coomassie blue binding by the GST portion of the fusion protein (C. Li and R.M. Evans, unpubl.).

ing to RXR can release corepressors from RAR and promote interactions with coactivators in vivo, the experiments illustrated in Figure 5 were carried out. Figure 5A shows that a VP16 activation domain-RXR ligand-binding domain fusion (VP16-RXR) does not interact with a GAL4 DNA-binding domain fusion to the receptor interacting domain of the silencing mediator SMRT (GAL4cSMRT; Chen and Evans 1995) when a two-hybrid assay is performed in CV1 cells. This observation confirms previous results indicating that RXR interacts weakly, if at all, with SMRT (Chen and Evans 1995; Chen et al. 1996). When VP16-RAR is included in the experiment along with VP16-RXR, however, significant activation of GAL4 reporter is observed in the absence of ligand (Figure 5B; open bar). As expected, addition of the RARspecific ligand AM580 disrupts the RAR-SMRT interaction leading to a decrease in reporter activity of $>95 \%$ (hatched bar). Similarly, LG100754 leads to a decrease in reporter activity of $\sim 80 \%$ (solid bar). In contrast, addition of the RXR-specific ligand LGD1069 displays only a modest effect (shaded bar). The failure to detect an interaction between RXR and SMRT (Fig. 5A) suggests that binding of LG100754 to RXR produces a conformational change in RAR that promotes dissociation of SMRT (Fig. 5B). To ensure the ability of LG100754 to promote release of SMRT from RAR was dependent upon RXR, the SMRT-RAR two-hybrid experiment was repeated in Saccharomyces cerevisiae, an organism without endogenous RXRs. Figure $5 \mathrm{E}$ shows that in the absence of RXR, LG100754 fails to promote release of SMRT.

To determine whether binding of LG100754 to RXR can promote an association between RAR and a positively acting cofactor, the interaction between RAR and the recently identified coactivator SRC-1 (Oñate et al. 1995) was examined. Although several receptors including RAR have been shown to interact with a carboxyterminal domain of SRC-1 (Kamei et al. 1996; Oñate et al. 1995; I.G.S. unpubl.), the two-hybrid assay shown in Figure 5C indicates that RXR alone does not interact with this domain of SRC-1. When VP16-RAR is cotransfected along with VP16-RXR (Fig. 5D), however, a strong interaction is detected in the presence of LG100754 (solid bar). The effect of LG100754 is similar to that observed with the RAR-specific ligand AM580 (hatched bar). Addition of the RXR-specific ligand LGD1069 does not promote an interaction with the carboxy-terminal domain of SRC-1 (shaded bar). Once again, a two-hybrid experiment in $S$. cerevisiae indicates that RXR is required for LG100754 to promote an interaction between RAR and SRC-1 (Fig. 5F). The ability of LG100754 to promote an interaction between RAR and SRC-1 and at least partially disrupt the RAR-SMRT interaction further supports the hypothesis that binding of this ligand to RXR allows activation of transcription via the RAR $\tau \mathrm{c}$ domain.

\section{Discussion}

The ability of small molecules to regulate transcription by directly binding to and modulating the activity of transcription factors was first proposed by Jacob and Monod over 30 years ago from studies on enzyme induction in bacteria (Jacob and Monod 1961). The results of the present study on ligand-dependent trans-activation by nuclear hormone receptors identify a unique form of allosteric control of transcription by small molecules. As shown in the model depicted in Figure 6, the data suggest that binding of the novel RXR-specific ligand LG100754 to one subunit, RXR, of an RXR-RAR heterodimer results in a conformational change in the noncovalently bound second subunit RAR. This conformational change results in both the dissociation of corepressors and association of coactivators in a fashion mediated by the activation function ( $\tau \mathrm{c}$ domain) of the nonliganded RAR subunit. Thus, although LG100754 binds to RXR, it is RAR that behaves as if it is liganded. Preliminary experiments suggest that activation of RXR-PPAR heterodimers occurs by a similar mechanism. We have 

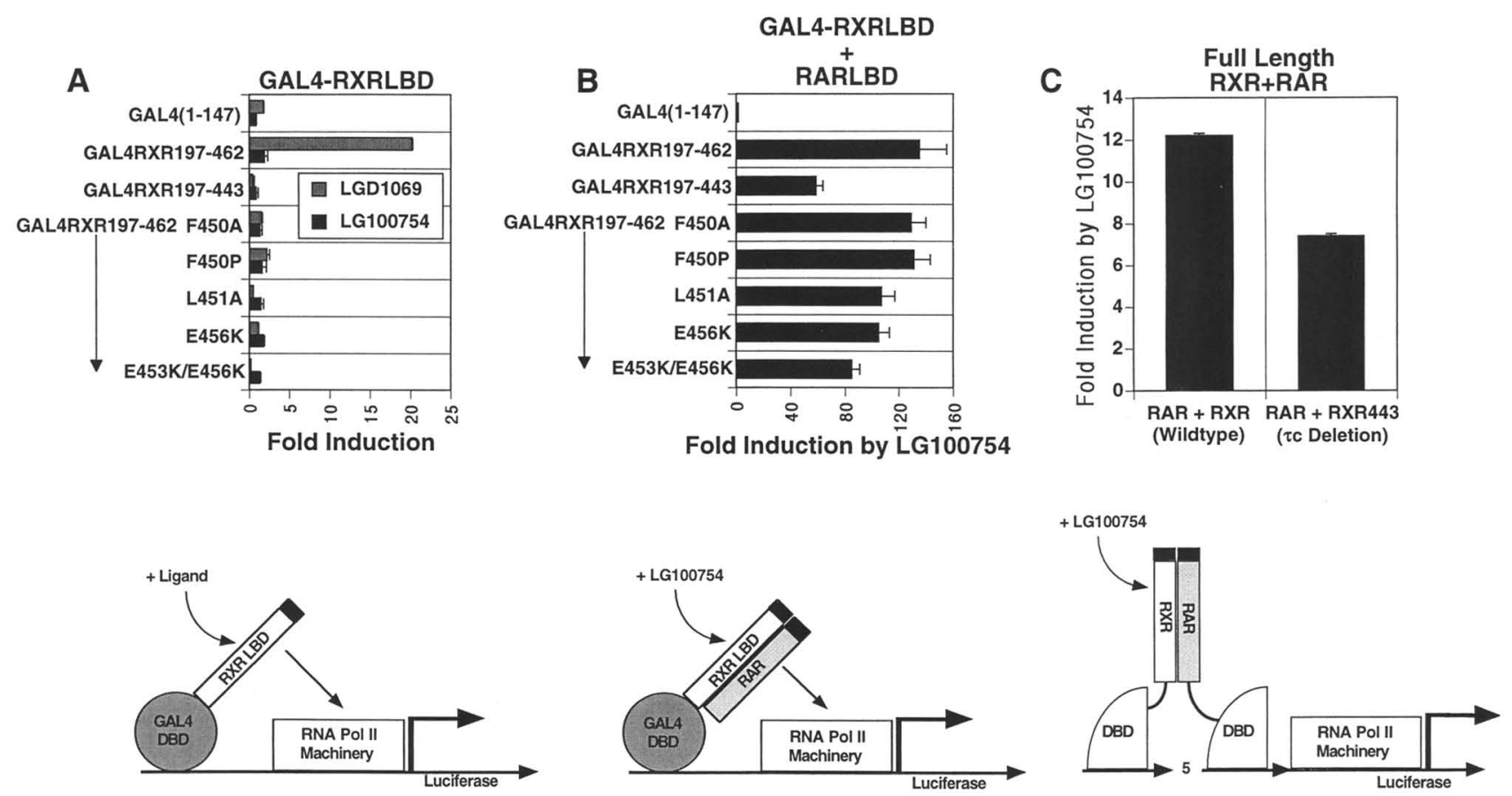

Figure 3. The RXR $\tau c$ domain is not required for LG100754-mediated trans-activation. (A) Fusions between the DNA-binding domain of GAL4 and RXR ligand binding domain (amino acids 197-462 or 197-443) were transfected into CV1 cells along with a reporter with 4 GAL4 binding sites $\left(\mathrm{UAS}_{\mathrm{G}} \mathrm{X}\right.$ 4). The $\tau \mathrm{c}$ domain deletion mutant consists of amino acids 197-443. Point mutants consist of amino acids 197-462 of RXR. After transfection, CV1 cells were cultured in the presence or absence of 100 nM LGD1069 (shaded bars) or $100 \mathrm{nM}$ LG100754 (solid bars) for $36 \mathrm{hr}$. Fold induction by ligand (+ligand/-ligand) is presented. The schematic underneath the graph illustrates the GAL4-based system used. (B) Fusions between the DNA binding domain of GAL4 and RXR ligand binding domain (amino acids 197-462 or 197-443) were transfected into CV1 cells along with a construct expressing the ligand-binding domain of human RAR $\alpha$ and a reporter with 4 GAL4-binding sites $\left(\mathrm{UAS}_{\mathrm{G}} \times 4\right)$. The $\tau \mathrm{c}$ mutants are the same described in $A$. After transfection, CV1 cells were cultured in the presence or absence of $100 \mathrm{nM}$ LG100754 for $36 \mathrm{hr}$. Fold induction by LG100754 (+LG100754/-LG100754) is presented. The schematic underneath the graph illustrates heterodimer formation between the GAL4-RXR fusions and the RAR ligand-binding domain. $(C)$ Constructs expressing full-length human RAR $\alpha$ a were cotransfected into CV1 cells along with constructs expressing full length human RXR $\alpha$ (RXR) or the RXR $\tau c$ domain deletion (RXR443). A luciferase reporter containing two copies of synthetic direct repeats spaced by 5 (DR5 $\times 2$-luc) was also included in the transfections. Cells were treated as described in $B$. The schematic underneath the graph illustrates heterodimer formation between the full length RXR and RAR. Transfection results in $A-C$ were normalized by cotransfection with a $\beta$-galactosidase expression plasmid (see Materials and Methods).

termed this unique form of allosteric control the phantom ligand effect.

How does LG100754 binding to RXR lead to transactivation by RAR? Clearly binding of LG100754 must result in some type of conformational change in the RXR LBD. This hypothesis is supported by the recent data of Lala et al. (1996) who showed that LG100754 induces a conformational change in RXR /detected by interaction with TAF110) but does not allow the RXR $\tau$ c domain to achieve an active conformation (detected by the failure of RXR homodimers to interact with TBP). The inability of LG100754 to promote interactions between RXR and coactivators likely accounts for the ability of LG100754 to act as an antagonist of RXR homodimers (Lala et al. 1996). Thus, the unique properties of LG100754 appear to arise from combining the qualities of both agonists and antagonists into one molecule.

Interestingly, Lala et al. (1996) were unable to observe LG100754-dependent release of SMRT from RAR in vitro. The discrepancy between the in vitro data of Lala et al. and the in vivo data (two-hybrid experiment; Fig. 5) presented in this work most likely arises from a difference in the sensitivity of the two assays. We would predict that with a relatively weak agonist like LG100754, RAR would be in a dynamic equilibrium shifting between inactive (SMRT bound) and active (SMRT released) conformations. The gelshift experiment is a biochemical assay with a single fixed point. This in vitro assay also requires a large excess of SMRT relative RXRRAR in order to observe the RAR-SMRT interaction. This high ratio of SMRT to RXR-RAR most likely requires RAR to be locked in an active conformation in order to observe dissociation of SMRT. In subsequent gel-shift assays in which the SMRT to heterodimer ratio has been modified, we have observed $20 \%-30 \%$ SMRT dissociation by LG100754 (data not shown). On the other hand, the two-hybrid assay is integrated over time and the ratio of SMRT to RXR-RAR is closer to $1: 1$. This more physiological ratio of SMRT to RXR-RAR, along with the dynamic nature of the assay, most likely ren- 


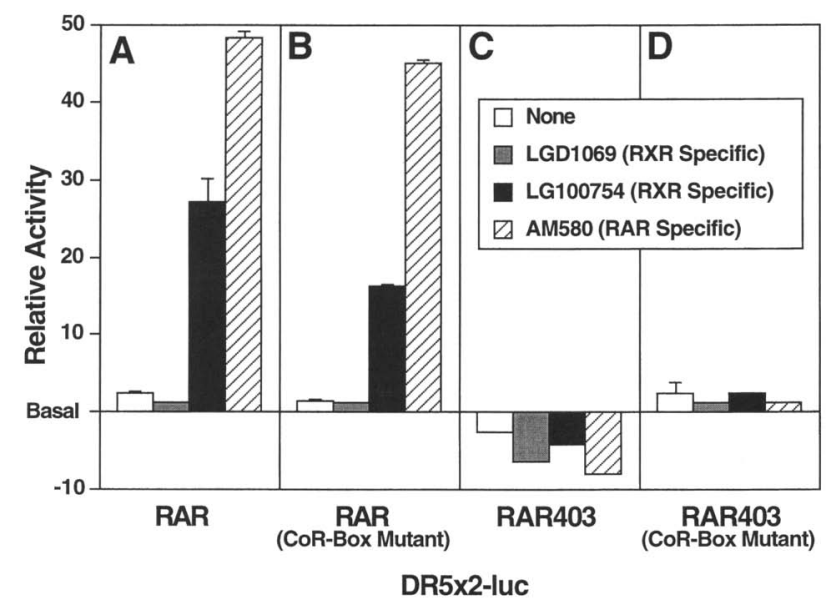

Figure 4. The RAR $\tau c$ domain is essential for LG100754-mediated trans-activation. Constructs expressing full length human $\operatorname{RAR} \alpha(A)$, the full-length RAR CoR-box mutant $(B)$, the RAR $\tau \mathrm{c}$ domain deletion (RAR403; C), or the RAR CoR-box/ $\tau \mathrm{c}$ domain deletion $(D)$ were transfected into CV1 cells. A luciferase reporter containing two copies of synthetic direct repeats spaced by 5 (DR $5 \times 2-$ luc) was also included in the transfections. CV1 cells were cultured in the absence (open bars) or presence of $100 \mathrm{~nm}$ LGD1069 (shaded bars), LG100754 (solid bars) or AM580 (hatched bars) for $36 \mathrm{hr}$. Activity relative to the DR5 $\times 2$-luciferase reporter alone is reported. In this experiment, endogenous RXR provides the dimerization partner for transfected RAR. Cotransfection of an RXR expression plasmid along with the RAR expression plasmids yields similar results (data not shown). Transfections were normalized by cotransfection with a $\beta$-galactosidase expression plasmid (see Materials and Methods).

ders the two-hybrid experiment more sensitive to subtle changes in the RAR-SMRT interaction. Also, the possibility that additional trans-acting factors are required for LG100754-dependent release of SMRT cannot be ruled out. Nevertheless, even in the two-hybrid experiment we can detect a difference of almost two orders of magnitude when SMRT dissociation by LG100754 is compared with dissociation by RAR-specific agonists such as AM580.

Binding of ligand by nuclear hormone receptors is thought to initiate a $180^{\circ} \mathrm{C}$ flip in the omega loop connecting helices 1 and 3 of RAR (helices 2 and 3 of RXR) and a $90^{\circ}$ angular shift in the $\tau \mathrm{c}$ domain (helix 12). These changes are believed to result in a conformation that promotes association with positive coactivators and/or components of the basal transcription machinery as well as dissociation of trans-acting corepressors (for review, see Mangelsdorf and Evans 1995; Schwabe 1996; Wurtz et al. 1996). Activation by the RAR $\tau c$ domain after binding of ligand to RXR suggests that an altered RXR structure triggers a similar change in RAR. It seems inescapable that this change must be propagated through the heterodimerization interface (helices 9 and 10). This, in turn, has two implications. First, ligand binding not only changes the omega loop and $\tau c$ domain but also alters the dimer interface. Second, the dimer interfaces are linked such that a change in one (i.e., RXR) results in a consequential linked change in the partner. This change is then transmitted throughout the non-liganded LBD to the $\tau c$ domain resulting in a conformation that mimics ligand binding even in the absence of bound ligand. Thus, it is more appropriate to consider RXR-dependent heterodimers as structural and functional units. Accordingly, the DNA-binding, ligand-binding, repression, and trans-activation properties are products of the complex and are not simply the sum of two independent subunits.

\section{The phantom ligand effect}

The concept of the phantom ligand derives from the observation that the RAR $\tau c$ domain is essential for the response to LG100754 even though RAR itself is unoccupied (Fig. 3). Although deletion of the RAR $\tau \mathrm{c}$ domain produces a dominant negative receptor with high affinity for corepressors (Baniahmad et al. 1992; Damm et al. 1993; Chen and Evans 1995; Schulman et al. 1996), the requirement for the RAR $\tau c$ domain is still observed when corepressor binding is reduced by mutation of the CoR-box. The requirement for the RAR $\tau c$ domain in the absence of repression favors the hypothesis that RAR contributes to positive trans-activation by LG100754 and is not only involved in relief of repression. It should be pointed out, however, that the RAR403-CoR-box mutant still binds SMRT at a reduced level $(-10 \%$ of wildtype RAR). Thus, the possibility that corepressor binding masks LG100754-mediated trans-activation by a unique activation surface on RXR cannot be ruled out completely. Nevertheless, the ability of LG100754 binding to RXR to dissociate SMRT from RAR and promote an interaction between RAR and SRC-1 indicates that the RAR $\tau c$ domain must be undergoing a conformational change. Finally, although LG100754 is not significantly metabolized during the course of a transfection experiment, we cannot rule out the possibility that in vivo LG100754 can bind directly to RAR.

Recent studies on members of the steroid and thyroid nuclear hormone receptor superfamily have identified other types of allosteric effects on transcription. Analysis of the glucocorticoid receptor has suggested that DNA binding can allosterically regulate trans-activation (Lefstin et al. 1994; Starr et al. 1996) and Forman et al. (1995) have shown that dimerization with nonpermissive partners can allosterically affect ligand binding by RXR. Also, high affinity ligand binding by the Drosophila ecdysone receptor requires dimerization with the RXR homolog Ultraspiracle (Yao et al. 1993). This is the first report to show that binding of ligand by one receptor leads to activation of transcription mediated by the nonliganded partner. The observations made with the synthetic ligand LG100754 indicate a remarkable flexibility in the ability of heterodimeric nuclear receptors to respond to ligand and suggest the possibility that naturally occurring phantom ligands may exist in vivo. Thus the phantom ligand effect has multiple implications with regard to the mechanism of hormone action. In addition, the ability to regulate hormonal signaling alloste- 
CV1 (+RXR)

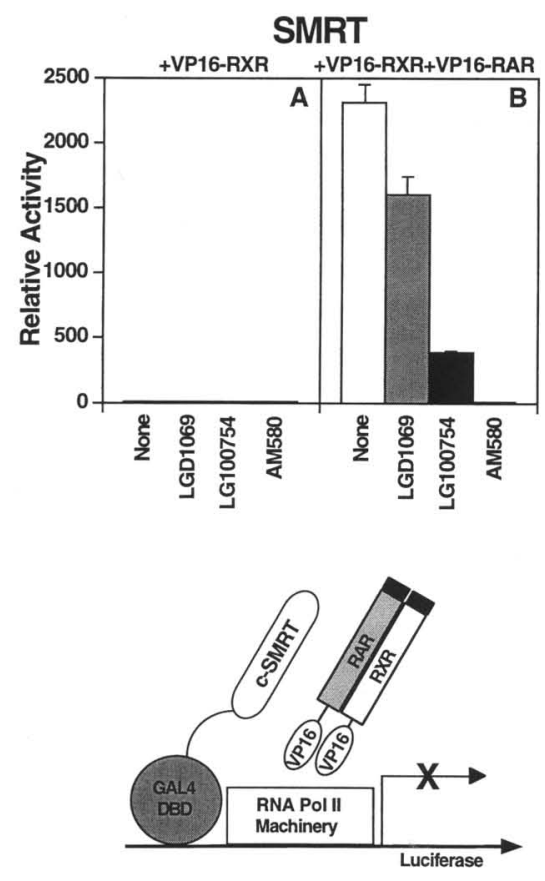

SRC-1
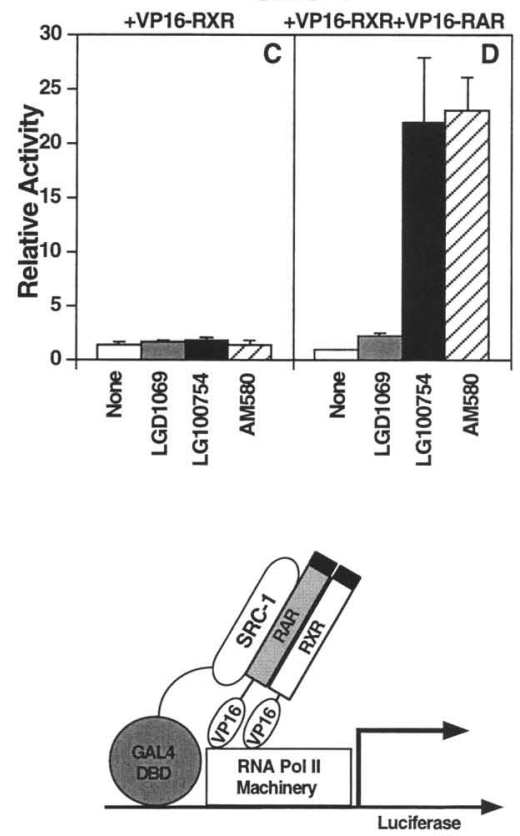

Yeast (-RXR)
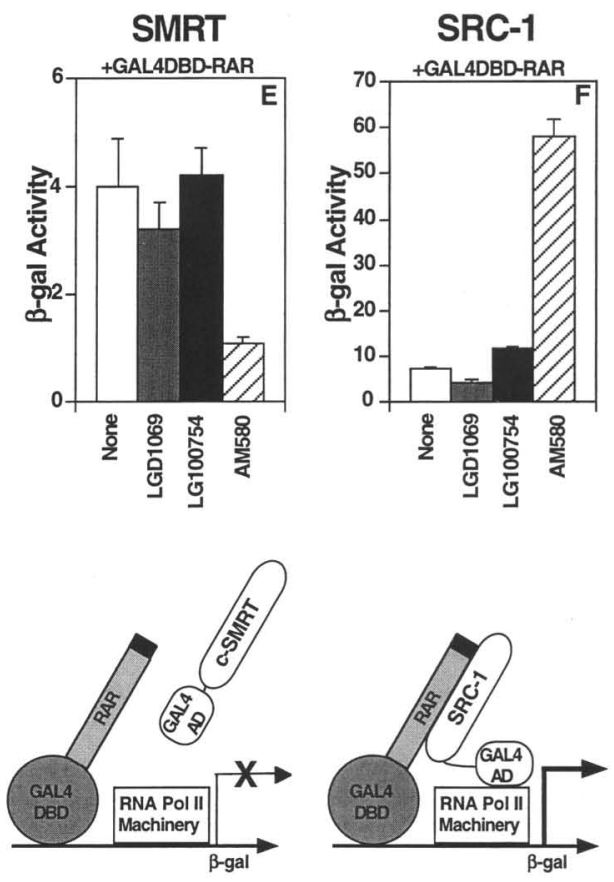

Figure 5. LG100754 dissociates SMRT and promotes an interaction between SRC-1 and RAR. (A) A fusion between the DNA-binding domain of GAL4 and the receptor interacting domain of SMRT (GAL4-cSMRT) was cotransfected into CV1 cells along with a construct expressing a VP16 activation domain-human RXR $\alpha$ ligand-binding domain (VP16-RXR) fusion protein. A luciferase reporter with 4 GAL4-binding sites $\left(\mathrm{UAS}_{\mathrm{G}} \times 4\right.$-luc) was also included. CV1 cells were cultured in the absence (open bars) or presence of $100 \mathrm{nM}$ LGD1069 (shaded bars), LG100754 (solid bars) or AM580 (hatched bars) for $36 \mathrm{hr}$. Activity relative to GAL4-cSMRT alone is reported. (B) Same as $A$; however, a construct expressing a VP16 activation domain-human RAR $\alpha$ ligand-binding domain (VP16-RAR) fusion protein was also included. (C) A fusion between the DNA-binding domain of GAL4 and the carboxy-terminal receptor interacting domain of SRC-1 (GAL4-SRC-1C; amino acids 940-1061 of the partial human SRC-1 identified by Oñate et al. 1995) was cotransfected into CV1 cells along with a construct expressing a VP16 activation domain-human RXR $\alpha$ ligand-binding domain (VP16-RXR) fusion protein. A luciferase reporter with 4 GAL4-binding sites $\left(\mathrm{UAS}_{\mathrm{G}} \times 4\right.$-luc $)$ was also included. CV1 cells were cultured in the absence (open bars) or presence of $100 \mathrm{nM}$ LGD1069 (shaded bars), LG100754 (solid bars) or AM580 (hatched bars) for 36 hr. Activity relative to GAL4-SRC-1 alone is reported. (D) Same as $C$; however, a construct expressing a VP16 activation domain-human RAR $\alpha$ ligandbinding domain (VP16-RAR) fusion protein was also included. Transfections were normalized by cotransfection with a $\beta$-galactosidase expression plasmid (see Materials and Methods). (E) The yeast strain Y190 was transformed with constructs expressing a GAL4 DNA-binding domain- human RAR $\alpha$ ligand-binding domain fusion (pG6H-RARLBD) and a GAL4 activation domain-cSMRT fusion (pACTII-cSMRT). $\beta$-Galactosidase activity was determined after $16 \mathrm{hr}$ of growth in the absence (open bars), or presence of $100 \mathrm{nM}$ LGD1069 (shaded bars), LG100754 (solid bars) or AM580 (hatched bars). (F) Same as E, however, pACTII-cSMRT was replaced with a construct expressing a GAL4 activation domain-SRC-1 fusion (amino acids 940-1061 of the partial human SRC-1 identified by Oñate et al. 1995).

rically through RXR indicates that it may be possible to regulate previously independent signaling pathways such as RXR-RAR and RXR-PPAR with a single ligand. Given the multiple contributions of heterodimeric nuclear hormone receptors to vertebrate development, human malignancies, and metabolic diseases, heterodimer-specific compounds such as LG100754 are likely to provide new insight into how nuclear hormone receptors influence these processes.

\section{Materials and methods}

\section{Plasmids}

Constructs expressing full-length human RXR $\alpha$, the RXR $\tau c$ domain deletion (RXR443), the GAL4-RXR ligand-binding do- main fusion (amino acids 197-462), the GAL4-RXR $\tau \mathrm{c}$ domain deletion (amino acids 197-443), the GAL4 DNA-binding domain SMRT receptor interacting domain fusion (GAL4cSMRT; amino acids 995-1495 of SMRT), full-length human RAR $\alpha$, and the human RAR $\alpha$ ligand-binding domain were described previously (Umesono et al. 1991; Forman et al. 1995; Schulman et al. 1995; Chen et al. 1996; Schulman et al. 1996). The VP16-RAR and VP16-RXR ligand-binding domain fusions contain the 78 amino acid trans-activation domain of VP16 from pVP16C1 (Novagen) fused to glutamic acid 156 of human RAR $\alpha$ or glutamic acid 203 of human RXR $\alpha$. Both fusions were cloned into pCMX (Umesono et al. 1991). Plasmids expressing the SRC-1 carboxy-terminal domain /GAL4-SRC-1C; amino acids 940-1061 of the partial human SRC-1 identified by Oñate et al. 1995) fused to the GAL4 DNA-binding domain [pCMXGAL4-SRC1-(940-1061)] and the GAL4 activation domain 

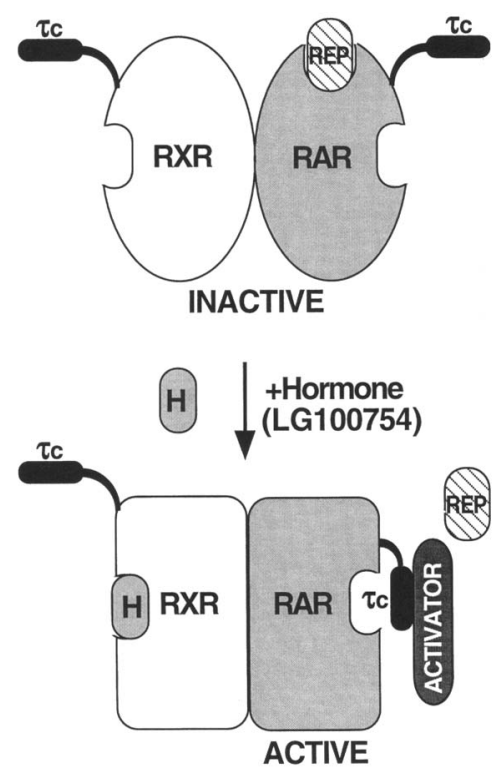

Figure 6. Allosteric transactivation by RXR. Binding of LG100754 by RXR induces a structural change in RXR-RAR heterodimers that allows the release of corepressors and the RAR $\tau$ c domain to achieve an active conformation. The change in shape upon binding of LG100754 by RXR signifies a conformational change in the heterodimer that results in movement of the RAR $\tau$ c domain toward the LBD core (see text for details).

[(pACTII-SRC1-(940-1061)] were provided by Ligand Pharmaceuticals. Point mutations were introduced into the RXR $\tau \mathrm{c}$ domain by PCR by use of oligonucleotides with the appropriate base changes. Amplified products were cloned into pCMXG4epi (Schulman et al. 1995). The RAR $\tau$ c domain deletion was constructed by PCR amplification of human RAR $\alpha$ (amino acids 1-403). A stop codon was introduced after position 403. The amplification product was cloned into HindIII-NheI digested pCMX. The RAR corepressor box mutants (CoR-box) were constructed by PCR amplification of human RAR $\alpha$ with three pairs of oligonucleotides. The first pair (R1 and $\mathrm{R} 2)$ were used to amplify amino acids 1-192. The two other pairs were used to amplify amino acids 184-462 (full length; R3 and R4) or amino acids 184-403 ( $\mathrm{rc}$ domain deletion; R3 and R5). Mutations encoding the following amino acid changes A194G, H195G, and T198A were incorporated into oligonucleotide R3. After amplification, the R1-R2 product was digested with HindIII and SacI. The R3-R4 and R3-R5 products were digested with SacI and NheI. The digested amplification products were ligated directly (R1-R2+R3-R4 and R1-R2+R3-R5) into HindIII-NheI-digested pCMX. For yeast two-hybrid assays, a GAL4 DNA-binding domain-RAR ligand-binding domain expression construct was generated by PCR amplification of the ligand-binding domain human RAR $\alpha$. The amplification product was cloned into pG6H (Schulman et al. 1995) as an NcoI-BgIII fragment. The GAL4 activation domain SMRT receptor interacting domain fusion has been described (Chen and Evans 1995). All PCR derived constructs were verified by sequencing. Luciferase reporters containing two copies of a synthetic direct repeat spaced by 5 nucleotides (DR5 $\times 2$-luc) or 4 GAL4-binding sites $\left(\mathrm{UAS}_{\mathrm{G}} \times 4\right.$ ) have been described previously (Forman et al. 1995; Umesono et al. 1991).

\section{Transfection}

CVl cells were plated in 48 -well plates at a density of $2 \times 10^{4}$ cells/well in DMEM supplemented with $10 \%$ charcoal-resin split fetal bovine serum. After growth at $37^{\circ} \mathrm{C}$ for $12-16 \mathrm{hr}$, cells were transfected with the DOTAP transfection reagent following the manufacturer's instructions (Boehringer Mannheim). For each well, $12 \mathrm{ng}$ of luciferase reporter, $36 \mathrm{ng}$ of the appropriate expression constructs and as an internal control, $60 \mathrm{ng}$ of pCMX-Bgal DNA was transfected. When necessary, the parental expression vector pCMX was added to ensure equivalent amounts of DNA were transfected in each well. After $5 \mathrm{hr}$ at $37^{\circ} \mathrm{C}$, the media was removed, the cells washed once and $200 \mu \mathrm{l}$ of fresh medium was added with or without the ligands described in the legend to each figure. Cells were harvested after 36 additional hr of growth at $37^{\circ} \mathrm{C}$. Luciferase activity of each sample was normalized by the level of $\beta$-galactosidase activity. Each transfection was carried out in duplicate and repeated at least three times.

\section{Purification of recombinant $R X R-R A R$ heterodimers}

The ligand binding domains of human RXR $\alpha$ and human RAR $\alpha$ were coexpressed in BL21 cells. The RXR ligand-binding domain had GST fused to amino terminus whereas the RAR ligand-binding domain had a 6 histidine tag at the amino terminus. The heterodimeric complex was purified by binding to $\mathrm{Ni}^{+2}$-NTA agarose (Qiagen Inc.) and eluted with a imidazole gradient. After elution from the $\mathrm{Ni}^{+2}$-NTA agarose, the pooled fractions were further purified by gel filtration on Superdex 200 (Pharmacia). The fractions that corresponded to the heterodimer as determined by the elution profile of known standards and SDS gel electrophoresis to ensure a 1:1 ratio of RXR to RAR were pooled and used in the ligand-binding experiments described below. The apparent excess of GST-RXR to 6 histidineRAR results from increased coomassie blue binding by the GST portion of the fusion protein. A detailed description of the purification and characterization of the recombinant heterodimer will be provided elsewhere (Li et al. 1997).

\section{Ligand binding and competition}

Two micrograms of RXR-RAR heterodimers (ligand-binding domains only/ purified as described above were resuspended in a final volume of $0.5 \mathrm{ml}$ binding buffer $(20 \mathrm{~mm}$ HEPES at $\mathrm{pH}$ $7.5 / 150 \mathrm{~mm} \mathrm{KCl} / 0.5 \%$ CHAPS). Cold ligands were added at a final concentration of $1.0 \mu \mathrm{M}$, immediately followed by the addition of ${ }^{3} \mathrm{H}$ all-trans retinoic acid at a final concentration of 1.0 nM. After addition of ligands, $20 \mu \mathrm{l}$ of a $1: 1$ slurry of $\mathrm{Ni}^{+2}$-NTA agarose was added and the samples were mixed gently for 60 min at room temperature to allow binding of the heterodimers to the agarose beads. The samples were then pelleted for $10 \mathrm{sec}$ at $15,000 \times \mathrm{g}$ and the supernatant was removed. The pellets were washed 3 times with $1.0 \mathrm{ml}$ of binding buffer, resuspended with $0.5 \mathrm{ml}$ of phosphate buffered saline, and transferred to a scintillation vial. After the addition of $10 \mathrm{ml}$ of Ecolum (ICN Biomedicals), the amount of ${ }^{3} \mathrm{H}$ all-trans retinoic acid bound was determined by scintillation counting. In the absence of added heterodimer $<50 \mathrm{cpm}$ was bound. Each sample was assayed in duplicate. For SDS gel analysis, samples treated in an identical fashion were resuspended with SDS gel sample buffer following the 3 post-binding washes, boiled and run on a $10 \%$ SDS gel.

\section{Yeast two-hybrid assay}

The strain Y190 (MATa gal4 gal80 his3 trp1-901 ade2-101 ura3 
52 leu2-3,-112 cyh ${ }^{\mathrm{r}}$ URA3::GAL1 $\rightarrow$ lacZ LYS2::GAL1 $\rightarrow$ HIS3| was used for all experiments. For $\beta$-galactosidase assays, a minimum of three independent transformants were grown overnight at $30^{\circ} \mathrm{C}$ in minimal media $(0.66 \%$ YNB, $2 \%$ glucose) supplemented with the appropriate amino acids. Cells were diluted 1:20 into fresh media and $100 \mathrm{nM}$ AM580, LGD1069, or LG100754 was added. $\beta$-Galactosidase activity was measured after $16 \mathrm{hr}$ of growth at $30^{\circ} \mathrm{C}$ as described by Rose et al. (1990).

\section{Acknowledgments}

We acknowledge S. Canan Koch (Ligand Pharmaceuticals) for providing LG100754 and communicating unpublished data on its metabolism and D. Lala and R. Heyman (Ligand Pharmaceuticals) for their advice and suggestions regarding the experiments described in this work. We also acknowledge the expert assistance of Henry Juguilon for help with transfections and Ester Banayo for purification of RXR-RAR heterodimers. Finally, we thank M. Manchester, D. Chakravarti, and B. Forman for their comments on the manuscript and B. Forman, D. Chakravarti, J. D. Chen (Salk Institute) for providing plasmids. The GAL4-SRC-1 plasmids were provided by Ligand Pharmaceuticals. I.G.S. was supported by a senior postdoctoral fellowship from the California division of the American Cancer Society and funds from the J. Aron foundation. J.W.R.S. was supported by the Human Frontier Science Program Organization. R.M.E. is an Investigator of the Howard Hughes Medical Institute at The Salk Institute for Biological Studies. This work was supported by grants GM 26444 and HD 27183 from the National Institutes of Health (R.M.E.).

The publication costs of this article were defrayed in part by payment of page charges. This article must therefore be hereby marked "advertisement" in accordance with 18 USC section 1734 solely to indicate this fact.

\section{References}

Allegretto, E.A., M.R. McClurg, S.B. Lazarchik, D.L. Clemm, S.A. Kerner, M.G. Elgort, M.F. Boehm, S.K. White, J.W. Pike, and R.A. Heyman. 1993. Transactivation properties of retinoic acid and retinoid $\mathrm{X}$ receptors in mammalian cells and yeast. Correlation with hormone binding and effects of metabolism. J. Biol. Chem. 268: 26625-26633.

Allenby, G., M.-T. Bocquel, M. Saunders, S. Kazmer, J. Speck, M. Rosenberger, A. Lovey, P. Kastner, J.F. Grippo, P. Chambon, and A.A. Levin. 1993. Retinoic acid receptors and retinoid X receptors: Interactions with endogenous retinoic acids. Proc. Natl. Acad. Sci. 90: 30-34.

Baniahmad, A., A.C. Kohne, and R. Renkawitz. 1992. A transferable silencing domain is present in the thyroid hormone receptor, in the v-erbA oncogene product and the retinoic acid receptor. EMBO J. 11: 1015-1023.

Bourguet, W., M. Ruff, P. Chambon, H. Gronemeyer, and D. Moras. 1995. Crystal structure of the ligand-binding domain of the human nuclear receptor RXR-alpha. Nature 375: 377382.

Canan Koch, S.S., L.J. Dardashti, J.J. Hebert, G.E. Croston, K.S. Flatten, R.A. Heyman, and A.M. Nadzan. 1996. Identification of the first retinoid $\mathrm{X}$ receptor homodimer antagonist. $J$. Med. Chem. 39: 3229-3234.

Cavailles, V., S. Dauvois, P.S. Danielian, and M.G. Parker. 1994. Interaction of proteins with transcriptionally active estrogen receptors. Proc. Nat1. Acad. Sci. 91: 10009-10013.
Chakravarti, D., V.J. LaMorte, M.C. Nelson, T. Nakajima, I.G. Schulman, H. Juguilon, M. Montminy, and R.M. Evans. 1996. Role of CBP/P300 in nuclear receptor signaling. $\mathrm{Na}$ ture 383: 99-103.

Chen, J.D. and R.M. Evans. 1995. A transcriptional co-repressor that interacts with nuclear hormone receptors. Nature 377: 454-457.

Chen, J.D., K. Umesono, and R.M. Evans. 1996. SMRT isoforms mediate repression and anti-repression of nuclear receptor heterodimers. Proc. Natl. Acad. Sci. 93: 7567-7571.

Claret, F.-X., T. Antakly, M. Karin, and F. Saatcioglu. 1996. A shift in the ligand responsiveness of thyroid hormone receptor a induced by heterodimerization with retinoid $\mathrm{X}$ receptor a. Mol. Cell. Biol. 16: 219-227.

Damm, K., R.A. Heyman, K. Umesono, and R.A. Evans. 1993. Functional inhibition of retinoic acid response by dominant negative retinoic receptor mutants. Proc. Natl. Acad. Sci. 90: 2989-2993.

Forman, B.M., K. Umesono, J. Chen, and and R.M. Evans. 1995. Unique response pathways are established by allosteric interactions among nuclear hormone receptors. Cell 81: 541-550.

Halachmi, S., E. Marden, G. Martin, H. MacKay, C. Abbondanza, and M. Brown. 1994. Estrogen receptor-associated proteins: Possible mediators of hormone induced transcription. Science 264: 1455-1458.

Hörlein, A.J., A.M. Naar, T. Heinzel, J. Torchia, B. Gloss, R. Kurokawa, A. Ryan, Y. Kamai, M. Soderstrom, C.K. Glass, and M.G. Rosenfeld. 1995. Hormone-independent repression by the thyroid hormone receptor is mediated by a nuclear receptor co-repressor N-COR. Nature 377: 397-404.

Jacob, F. and J. Monod. 1961. Genetic regulatory mechanisms in the synthesis of proteins. J. Mol. Biol. 3: 318-356.

Kamei, Y., L. Xu, T. Heinzel, J. Torchia, R. Kurokawa, B. Gloss, S.-C. Lin, R.A. Heyman, D.W. Rose, C.K. Glass, and M.G. Rosenfeld. 1996. A CBP integrator complex mediates transcriptional activation and AP-1 inhibition by nuclear receptors. Cell 85: 403-414.

Kastner, P., M. Mark, and P. Chambon. 1995. Nonsteroid nuclear receptors: What are genetic studies telling us about their role in real life? Cell 83: 859-869.

Kurokawa, R., J. DiRenzo, M. Boehm, J. Sugarman, B. Gloss, M.G. Rosenfeld, R.A. Heyman, and C.K. Glass. 1994. Regulation of retinoid signalling by receptor polarity and allosteric control of ligand binding. Nature 371: 528-531.

Kurokawa, R., M. Soderstrom, A. Horlein, S. Halachmi, M. Brown, M.G. Rosenfeld, and C.K. Glass. 1995. A co-repressor specifies polarity-specific activities of retinoic acid receptors. Nature 377: 451-457.

Lala, D.S., R. Mukherjee, I.G. Schulman, S.S. Canan-Koch, L.J. Dardashti, A.M. Nadzan, G.E. Croston, R.M. Evans, and R.A. Heyman. 1996. Activation of specific RXR heterodimers by an antagonist of RXR homodimers. Nature 383: $450-453$.

Leblanc, B.P. and H.G. Stunnenberg. 1995. 9-cis retinoic acid signaling: Changing partners causes some excitement. Genes \& Dev. 9: 1811-1816.

Lee, J.W., F. Ryan, J.C. Swaffield, S.A. Johnston, and D.D. Moore. 1995. Interaction of thyroid-hormone receptor with a conserved transcriptional mediator. Nature 374: 91-94.

Lefstin, J.A., J.R. Thomas, and K.R. Yamamoto. 1994. Influence of a steroid receptor-DNA-binding domain on transcriptional regulatory functions. Genes \& Dev. 8: 2842-2856.

Leng, X., J. Blanco, S.Y. Tsai, K. Ozato, B.W. O'Malley, and M.-J. Tsai. 1995. Mouse retinoid X receptor contains a separable ligand-binding and transactivation domain in its $\mathrm{E}$ region. Mol. Cell. Biol. 15: 255-263. 
Li, C., J.W.R. Schwabe, and R.M. Evans. 1997. Co-expression of nuclear receptor partners increases their solubility and biological activities. Proc. Natl. Acad. Sci. (in press).

Mangelsdorf, D.J. and R.M. Evans. 1995. The RXR heterodimers and orphan receptors. Cell 83: 841-850.

Mangelsdorf, D.J., C. Thummel, M. Beato, P. Herrlich, G. Schutz, K. Umesono, P. Kastner, M. Mark, P. Chambon, and R.M. Evans. 1995. The nuclear receptor superfamily: The second decade. Cell 83: 835-839.

Oñate, S.A., S.Y. Tsai, M.-J. Tsai, and B.W. O'Malley. 1995. Sequence and characterization of a coactivator for the steroid hormone receptor superfamily. Science 270: 1354-1347.

Renaud, J.-P., N. Rochel, M. Ruff, V. Vivat, P. Chambon, H. Gronemeyer, and D. Moras. 1995. Crystal structure of the RAR- $\gamma$ ligand-binding domain bound to all-trans retinoic acid. Nature 378: 681-689.

Rose, M.D., F. Winston, and P. Hieter. 1990. Methods in yeast genetics. Cold Spring Harbor Laboratory Press, Cold Spring Harbor, NY.

Schulman, I.G., D. Chakravarti, H. Juguilon, A. Romo, and R.M. Evans. 1995. Interactions between the retinoid X receptor and a conserved region of the TATA-binding protein mediate hormone-dependent transactivation. Proc. Natl. Acad. Sci. 92: 8288-8292.

Schulman, I.G., H. Juguilon, and R.M. Evans. 1996. Activation and repression by nuclear hormone receptors: Hormone modulates an equilibrium between active and repressive states. Mol. Cell. Biol. 16: 3807-3818.

Schwabe, J.W.R. 1996. Transcriptional control: How nuclear receptors get turned on. Curr. Biol. 6: 372-374.

Starr, D.B., W. Matsui, J.R. Thomas, and K.R. Yamamoto. 1996. Intracellular receptors use a common mechanism to interpret signaling information at response elements. Genes \& Dev. 10: 1271-1283.

Tate, B.F. and J.F. Grippo. 1995. Mutagenesis of the ligand binding domain of the human retinoic acid receptor a identifies critical residues for 9-cis-retinoic acid binding. I. Biol. Chem. 270: 20258-20263.

Tate, B.F., G. Allenby, R. Janocha, S. Kazmer, J. Speck, L.J. Sturzenbecker, P. Abarzua, A.A. Levin, and J.F. Grippo. 1994. Distinct binding determinants for 9-cis retinoic acid are located within AF-2 of retinoic acid receptor $\alpha$. Mol. Cell. Biol. 14: 2323-2330.

Umesono, K., K.K. Murakami, C.C. Thompson, and R.M. Evans. 1991. Direct repeats as selective response elements for the thyroid hormone, retinoic acid and vitamin $\mathrm{D}_{3}$ receptors. Cell 65: 1255-1266.

vom Baur, E., C. Zechel, D. Heery, M.J.S. Heine, J.M. Garnier, V. Vivat, B. Le Douarin, H. Gronemeyer, P. Chambon, and R. Losson. 1996. Differential ligand-dependent interactions between the AF-2 activating domain of nuclear receptors and putative transcriptional intermediary factors mSUG1 and TIF1. EMBO I. 15: 110-124.

Wagner, R.L., J.W. Apriletti, M.E. McGrath, B.L. West, J.D. Baxter, and R.J. Fletterick. 1995. A structural role for hormone in the thyroid hormone receptor. Nature 378: 690-697.

Wurtz, J.-M., W. Bourguet, J.-P. Renaud, V. Vivat, P. Chambon, D. Moras, and H. Gronemeyer. 1996. A canonical structure for the ligand-binding domain of nuclear receptors. Nature Struct. Biol. 3: 87-94.

Yao, T.P., B.M. Forman, Z. Jiang, L. Cherbas, J.D. Chen, M. McKeown, P. Cherbas, and R.M. Evans. 1993. Functional ecdysone receptor is the product of EcR and Ultraspiracle genes. Nature 366: 476-479. 


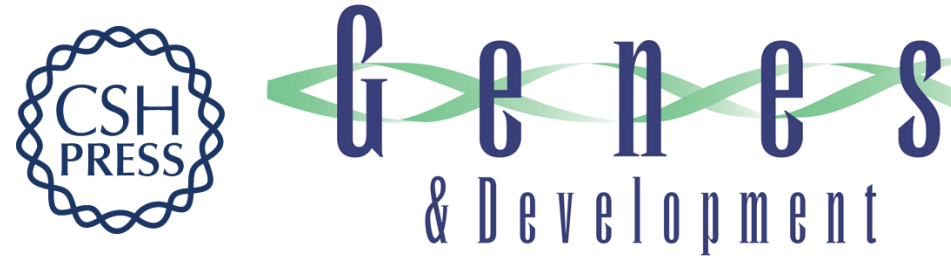

\section{The phantom ligand effect: allosteric control of transcription by the retinoid $X$ receptor.}

I G Schulman, C Li, J W Schwabe, et al.

Genes Dev. 1997, 11:

Access the most recent version at doi:10.1101/gad.11.3.299

References This article cites 39 articles, 16 of which can be accessed free at: http://genesdev.cshlp.org/content/11/3/299.full.html\#ref-list-1

License

Email Alerting

Service

Receive free email alerts when new articles cite this article - sign up in the box at the top right corner of the article or click here.

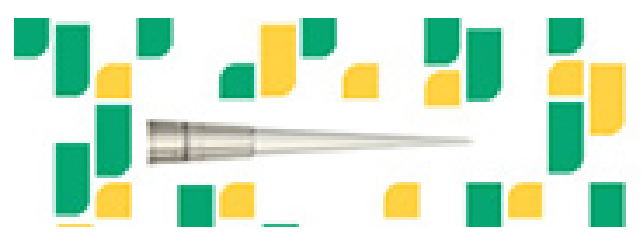

Focused on your science. 